\title{
Propagation characteristics of dust-acoustic waves in presence of a floating cylindrical object in the DC discharge plasma.
}

\author{
Mangilal Choudhary, ${ }^{1}$ a) S. Mukherjee, ${ }^{1}$ and P. Bandyopadhyay ${ }^{1}$ \\ Institute for Plasma Research, Bhat, Gandhinagar, 382428, \\ India
}

The experimental observation of the self-excited dust acoustic waves (DAWs) and its propagation characteristics in the absence and presence of a floating cylindrical object are investigated. The experiments are carried out in a direct current (DC) glow discharge dusty plasma in the background of argon gas. Dust particles are found levitated at the interface of plasma and cathode sheath region. The DAWs are spontaneously excited in the dust medium and found to propagate in the direction of ion drift (along the gravity) above a threshold discharge current at lower pressure. The excitation of such low frequency wave is a result of the ion-dust streaming instability in the dust cloud. The characteristics of the propagating dust acoustic wave get modified in presence of a floating cylindrical object of radius larger than the dust Debye length. Instead of propagating in the vertical direction, the DAWs are found to propagate obliquely in presence of the floating object (kept either vertically or horizontally). In addition, horizontally aligned floating object gives rise to form a wave structure in the cone shaped dust cloud in the sheath region. The change in the propagation characteristics of DAWs are explained on the basis of modified potential (or electric field) distribution, which is a consequence of coupling of sheaths formed around the cylindrical object and the cathode.

\footnotetext{
a) Electronic mail: mangilal@ipr.res.in
} 


\section{INTRODUCTION}

Dusty plasma is a complex system which is consisted of micron or sub-micron sized dust grains in the background of conventional two-component plasma. These particles are either formed as a result of agglomeration of reactive species or deliberately injected into the plasma. In low temperature plasma environment, highly mobile electrons resides on the dust surface and make these dust grains negatively charged up to $10^{3}-10^{5}$ times of an electronic charge $^{1}$. Above a critical density, these highly charged dust grains inside a plasma show collective nature similar to other plasma species (electrons and ions). Theoretical predication as well as experimental observation shows that the dusty plasma medium frequently supports the excitation of linear and nonlinear waves. Dust acoustic waves ${ }^{2-4}$, dust density waves ${ }^{5-7}$, dust acoustic transverse waves ${ }^{8,9}$, dust lattice waves ${ }^{10-12}$ are some of the linear modes whereas

dust acoustic solitary waves $^{13,14}$, dust ion acoustic shock waves ${ }^{15}$ and dust acoustic shock waves $^{16-18}$ are the nonlinear modes which have been studied broadly since last couple of decades.

After the invention of dust acoustic wave in $1995^{3}$, an extensive work (see the review paper by Merlino et al. ${ }^{19}$ ) on spontaneously excited DAWs has been carried out both theoretically and experimentally. Dust acoustic mode is an extremely low frequency compressive mode in which the dust particles provide the inertia and the shielding electron and ion clouds provide the thermal pressure effects. To understand the physics underlying the propagation characteristics of such low frequency modes, various experimental configurations have been employed to confine the dust particles ${ }^{20-25}$. It is experimentally shown in most of the studies that the self-excited dust acoustic waves in DC discharge dusty plasma are the consequence either of the streaming ions through dust $\operatorname{cloud}^{26,27}$ or dust charge variation in the confined dust $_{\text {cloud }}{ }^{25}$. Theoretical studies ${ }^{28-30}$ confirms that the spontaneous DAW gets triggered in most of the cases either by ions streaming or dust-charge fluctuation in the dust cloud which essentially supports the experimental observation.

The influence of a floating or biased object (more specifically the probe) on the confined dust cloud is an research area of current interest. In general, it is observed that a dust free region (called void) is formed around a floating object inserted inside a dust cloud in an anodic plasma ${ }^{31}$. The dust void around a negatively biased probe and its size dependence on the bias voltage is examined by Thomas et al. ${ }^{32}$. In the co-generated dusty plasma, a 
dust free region is formed around a ring shaped positively biased electrode ${ }^{33}$. Klindworth et al. ${ }^{34}$ studied the dust void around a Langmuir probe in a RF produced dusty plasma under microgravity conditions. Kim et al. ${ }^{35}$ experimentally investigated the dust acoustic wave which gets diffracted during the interaction with a floating cylinder. The experimental observation of the interaction of flowing dusty plasma with an electrically biased wire is studied by Meyer et al. ${ }^{36}$. In their study they found the floating wire excites the bow like shock structures. The effect of positively (or negatively) biased cylindrical probe on a strongly coupled dusty plasma (in crystalline state) is reported by Law et al. ${ }^{37}$. They observed that the biased probe, placed near the RF-sheath edge, alters the crystal properties around it and as a result a circulation of the dust particles is observed. The modification in the propagation characteristics of DAWs in presence of a floating object has not been explored much in these previous studies and is the subject matter of the present manuscript.

In this paper, we report an experimental observation of self-excited dust acoustic wave (DAWs) and its propagation characteristics in absence and presence of a floating cylindrical object in a DC glow discharge plasma. Similar to earlier observations, in absence of the rod, the dust cloud shows a spontaneous excitation of dust acoustic waves for a given discharge condition. The propagation characteristics of the dust acoustic waves are found to get significantly modified during the interaction with the floating rod. The modified characteristics of DAWs are explained on the basis of combined sheath electric field formed due to the presence of a floating object in the cathode sheath.

The manuscript is organized as follows: Sec. II deals with the detailed description on the experimental setup. Sec. III describes the plasma production and its characterization. A detailed description of dusty plasma production and its characterization is provided in Sec. IV. The experimental observation of dust acoustic waves and its characteristics is discussed in Sec. V. Sec. VI highlights the detailed characteristics of DAWs in presence of a floating rod. A brief summary of the work along with a concluding remarks is provided in Sec. VII.

\section{EXPERIMENTAL SETUP}

Experiments are performed in a borosilicate glass tube with an inner diameter of $15 \mathrm{~cm}$ and length of $60 \mathrm{~cm}$. The glass tube has 5 radial and 2 axial ports, used for pumping, 
gas feeding, plasma production and plasma/dusty plasma diagnostics purposes. A rotary pump and gas dosing valve are attached to a Stainless Steel (SS) buffer chamber of $30 \mathrm{~cm}$ length and $15 \mathrm{~cm}$ diameter, which is connected to the glass tube. The geometrical (3D) view of the experimental setup is described in more details elsewhere ${ }^{38}$. This experimental assembly prevents the direct gas flow induce neutral drag on the dust particles in the main experimental chamber where the dusty plasma experiments are carried out. The schematic of operating configuration is presented in the Fig. 1. A SS cylindrical rod of $5 \mathrm{~mm}$ diameter is inserted in the experimental chamber over the cathode using a Wilson feedthrough. The experimental chamber is evacuated below $10^{-3}$ mbar pressure using the rotary pump and the argon gas is then fed into the chamber. The chamber is pumped down again to the base pressure. This process is repeated several times to reduce the impurities from the vacuum vessel. Finally the operating pressure is set to 0.07 mbar by adjusting the gas dosing valve and pumping speed.

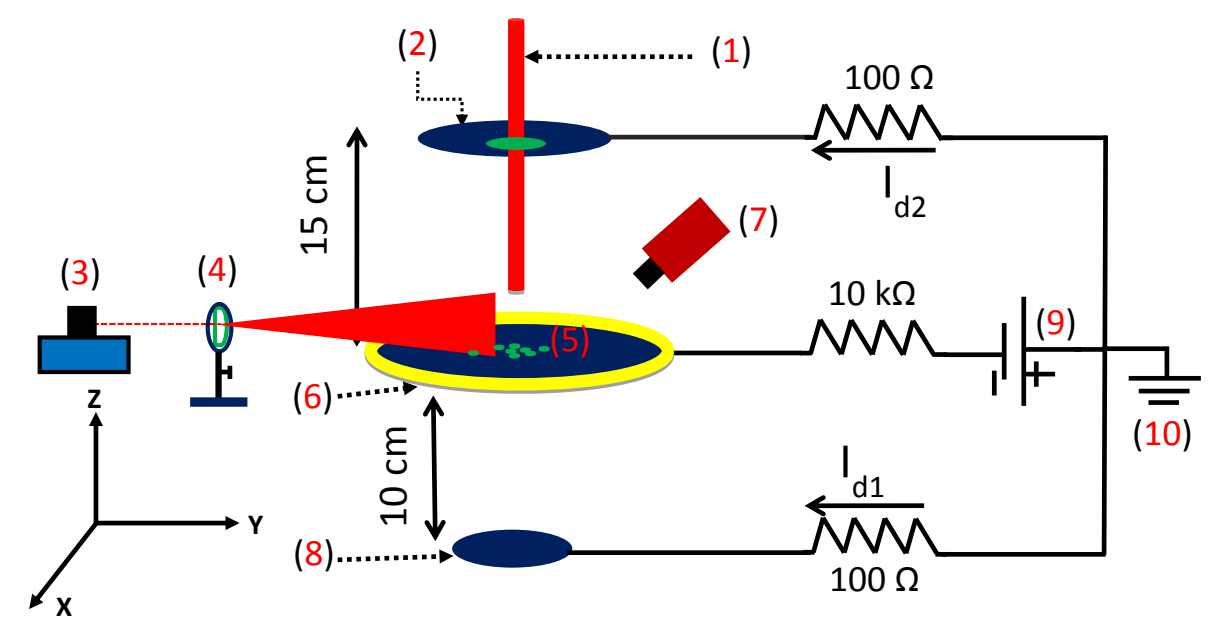

FIG. 1. Schematic of experimental configuration:(1) cylindrical rod. (2) Stainless steel(SS) flange (working as an anode). (3) solid-state red laser. (4) plano-convex cylindrical lens. (5) kaolin dust particles.(6) SS disk electrode (cathode) with a ring like step at corner (represented by yellow ring). (7) CCD camera. (8) Stainless Steel circular electrode (anode). (9) Discharge power supply $(1 \mathrm{kV}$ and $3 \mathrm{~A})$. (10) grounded. $I_{d 1}$ and $I_{d 2}$ represent the currents flowing in separate discharge path. 


\section{PLASMA PRODUCTION AND ITS CHARACTERIZATION}

A DC glow discharge plasma is initiated by applying a voltage in the range of 300-500 V in between a cathode and two anodes as shown in Fig. 1. The disk shaped cathode of $10 \mathrm{~cm}$ diameter is having plane surface in one side whereas the other side having a step of $5 \mathrm{~mm}$ width and $2 \mathrm{~mm}$ height around its periphery. The plane surface of the cathode is parallel to a SS disk of $4 \mathrm{~cm}$ in diameter, which is a one of the anodes (lower anode). This lower anode is kept $10 \mathrm{~cm}$ below from the cathode. Another anode (upper anode) is a SS flange of $7 \mathrm{~cm}$ diameter is parallel to the dust particles containing cathode surface. This upper anode is fixed $15 \mathrm{~cm}$ above the cathode. These above configuration of anodes and cathode demands minimum voltage to breakdown the argon gas. Both the anodes are kept grounded through a resistance of $R=100 \Omega$ to measure the discharge currents in each path. The experimental chamber is purposefully made of dielectric material and all the axial and radial ports are closed by toughened glasses and/or perspex flanges so that additional discharge paths can be avoided. There are some advantages to use this discharge configuration for performing dusty plasma experiments. In this present set of experiments, the dust particles are sprinkled homogeneously over the cathode surface which acts as a dielectric covered electrode. For breaking down the gas, cathode electrode is made negatively biased with respect to the grounded anodes. In absence of lower anode, 30-40 V more bias voltage is required to break down the gas and as a result it becomes difficult to form an appropriate dust cloud at lower gas pressure.

The variation of discharge current $\left(I_{d}\right)$ in both the discharge paths with discharge voltage $\left(V_{d}\right)$ are measured in absence of dust particle. The $I_{d}-V_{d}$ curves at pressure $\mathrm{p}=0.07$ mbar are displayed in Fig. 2(a). It is clearly seen in Fig. 2(a) that at first, $I_{d 1}$ (represented by closed rectangular) starts flowing at $320 \mathrm{~V}$ whereas $I_{d 2}$ (represented by closed circle) is initiated at $340 \mathrm{~V}$. It indicates that the plasma initially forms in between the cathode and lower anode at about $320 \mathrm{~V}$ and later it strikes between the cathode and the upper anode. It is also to be noted that $I_{d 1}$ stays always higher than the $I_{d 2}$ for the whole range of discharge voltage. It is also worth mentioning that at a particular discharge condition, the flowing currents $\left(I_{d 1}\right.$ and $\left.I_{d 2}\right)$ in each path depend upon the dimension of each electrodes.

The plasma parameters at various discharge conditions are measured using a cylindrical Langmuir probe of length $7 \mathrm{~mm}$ and radius of $0.25 \mathrm{~mm}$. The variations of plasma param- 
eters with discharge voltage are displayed in the Fig. 2(b). The plasma density $(n)$ and the electron temperature $\left(T_{e}\right)$ change from $\sim 1 \times 10^{9}$ to $7 \times 10^{9} \mathrm{~cm}^{-3}$ and $\sim 2.5$ to $5 \mathrm{eV}$, respectively with the change of discharge voltage from 380 to $540 \mathrm{~V}$ at a constant pressure $\mathrm{p}=0.07$ mbar. The measured values shows that the plasma density increases whereas the electron temperature decreases with the increase of discharge voltage. This essentially signifies that with the increase of discharge voltage the electron Debye length $\left(\lambda_{e}=\sqrt{\epsilon_{0} k T_{e} / n e^{2}}\right)$ decreases. The ions are assumed at room temperature i.e. $T_{i} \sim 0.025 \mathrm{eV}$. At a given discharge condition, these plasma parameters (measured outside the cathode sheath region) are used to calculate the dusty plasma parameters.

\section{DUSTY PLASMA AND ITS CHARACTERIZATION}

In course of dusty plasma experiments, kaolin dust particles with mass density $(\rho)$ of 2.6 $\mathrm{gm} / \mathrm{cm}^{3}$ of radii $\left(r_{d}\right)$ ranging from 0.5 to $5 \mu \mathrm{m}$, are sprinkled over the cathode surface. For producing the dusty plasma, DC glow discharge is initiated between cathode and anodes at 0.07 mbar. The discharge current-voltage characteristics in presence of dust particles follow the similar behavior as shown in Fig 2 but with slightly lower values of discharge currents. In presence of the plasma, the dust particles get negatively charged because of impinging of more electrons than ions on the surface of dust particles. The negatively charged dust particles lifted up from the cathode surface and levitate at the interface of plasma and cathode sheath region. The cathode geometry (ring at its periphery)provides the better radial confinement to the levitated dust particles. The levitated dust cloud is then illuminated by a red laser of $632 \mathrm{~nm}$ wavelengths and power ranges from 1-100 $\mathrm{mW}$.

The point laser source is converted into a laser sheet using a plano-convex cylindrical lens of focal length of $25 \mathrm{~mm}$ which can be oriented in vertical as well as horizontal plane. This laser sheet is efficient enough to illuminate $\sim 2 \mathrm{~mm}$ wide vertical (or horizontal) slice of the dust cloud. Due to the poly-dispersive nature of the particles, the heavier dust particles levitate at the bottom whereas the lighter particle levitate at the top of the sheath by balancing the electrostatic force and the gravitational force. The dynamics of the particles in vertical as well as in horizontal plane are recorded at 130 frames per second (fps) by a Lumenera CCD camera with the resolution 1088 pixels $\times 2048$ pixels. The data is then transfer to a high speed computer for the purpose of further analysis using different softwares. 

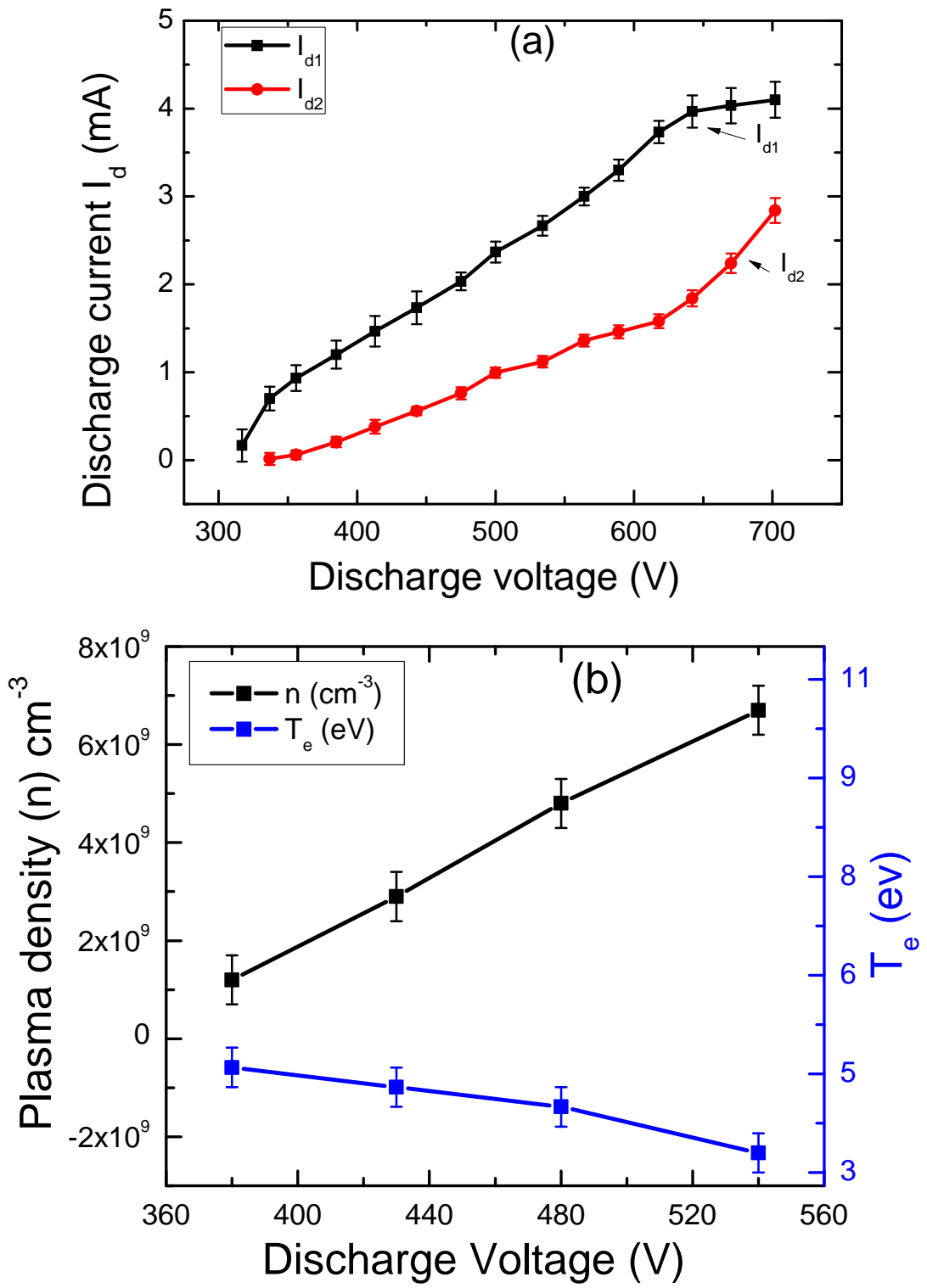

FIG. 2. (a) Discharge current $\left(I_{d 1}\right.$ and $\left.I_{d 2}\right)$ variation with discharge voltage (between cathode and anodes). Discharge currents are computed by dividing the voltage drop across a resistance of 100 $\Omega$. (b) Variation of plasma density $(n)$ and electron temperature $\left(T_{e}\right)$ with the discharge voltage. The argon pressure is set to 0.07 mbar

The effect of discharge parameters on the levitated dust cloud is also necessary to understand the propagation characteristics of dust acoustic waves. Therefore the sheath thickness and the width of the dust cloud are measured at given pressure for various discharge voltages. The sheath thickness is estimated as the distance between the cathode and the topmost layer of the dust particles whereas the difference of topmost and the bottommost layers gives the 
width of the dust cloud. The sheath thickness is found to decrease with the increase of the applied discharge voltage as shown in Fig. 3 (represented by solid rectangular). This experimental observations is in accordance with the theoretical estimation. According to Child-Langmuir law, the high voltage cathode sheath is function of the plasma Debye length (in general sheath thickness is ten to hundred times more than the Debye length ${ }^{39}$ ). As mentioned earlier, with the increase of discharge voltage the electron Debye length decreases which results in the decrease of sheath thickness. As sheath thickness reduces, the electric field gradient at sheath edge increases therefore the dust cloud get suppressed. The variation of width of the dust cloud with discharge voltage is also shown in Fig. 3 (represented by solid sphere).

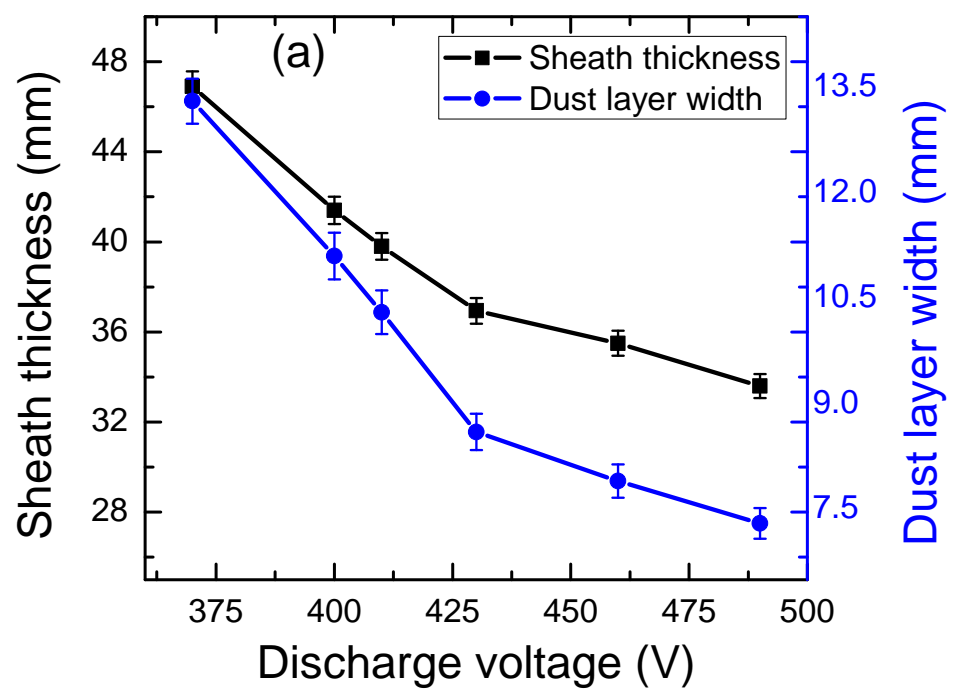

FIG. 3. The variation of sheath thickness (solid rectangular) and dust cloud width (solid sphere) with applied discharge voltages. The argon filling pressure during the experiment is set at 0.07 mbar.

\section{EXCITATION AND PROPAGATION CHARACTERISTICS OF DUST ACOUSTIC WAVES}

The dust particles form a steady state equilibrium approximately at $40-45 \mathrm{~mm}$ above the cathode at $V_{d}=340 \mathrm{~V}$ and $p=0.07$ mbar. If the discharge voltage is increased slightly to $\sim 350 \mathrm{~V}$, an appearance of spontaneous Dust Acoustic Wave (DAW) is found. This wave originates in the upper part of the levitated dust cloud and propagates towards the 
cathode (along the gravity). A typical video image of this self-excited DAWs (in $\mathrm{Y}-\mathrm{Z}$ plane) at $V_{d}=380 \mathrm{~V}$ and $p=0.07$ mbar is presented in Fig. 4(a). The stable dust cloud shows the excitation of spontaneous dust acoustic wave when the ratio $E / p$ crosses a threshold value ${ }^{26}$. This ratio increases either with the increase of electric filed (or the discharge voltage) or decrease of gas pressure. At higher pressure and higher discharge voltage, the wave could not excites due to frequent ion-neutral collision. Whereas at higher discharge voltage and lower pressure, the dust acoustic wave gets highly unstable ${ }^{23}$. Hence keeping both the points into mind, a constant pressure at $\mathrm{p}=0.07$ mbar is chosen to perform the experiments at a range of discharge voltage. The average intensity profile of Fig. 4(a) is shown in the Fig. 4(b). It is observed in the figure that the wave initially gets excited with smaller amplitude on the top and then propagates with increasing amplitude. It happens due to the non-uniformity of electric field inside the cathode sheath. The ions initially enter into the sheath with Bohm velocity and then accelerate towards the cathode and as a result these ions exert more pressure on the dust particles when they reach towards the cathode. It results in the increase of amplitude of DAWs while they propagate towards the cathodes. For the detailed characterization of DAWs, few consecutive frames are considered. The time

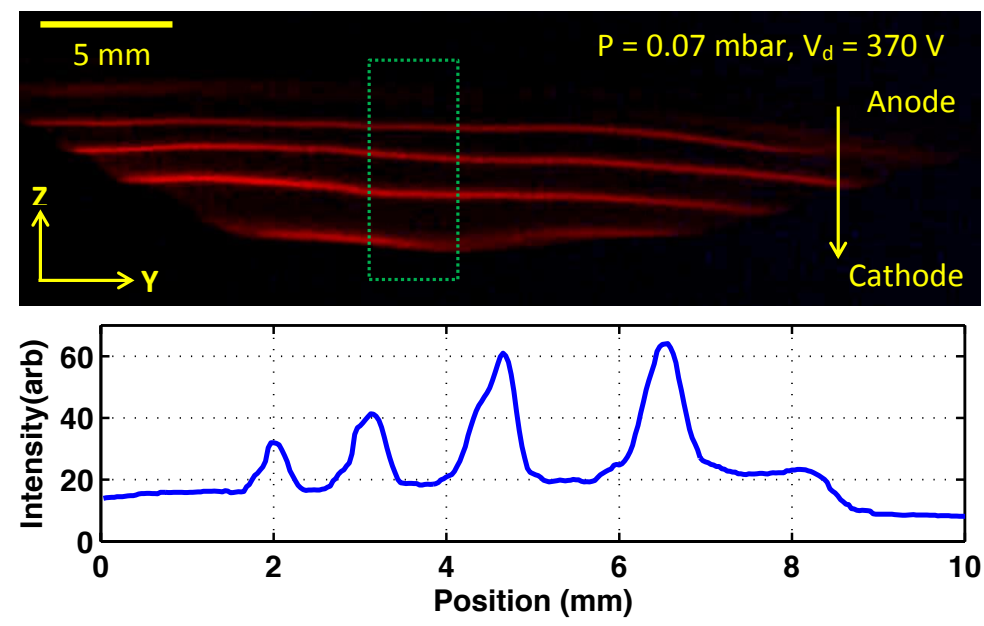

FIG. 4. (a) A video image of Dust Acoustic Waves in vertical (Y-Z) plane. Arrow indicates the velocity vector for DAW from anode to cathode. (b) Average intensity profile of scattered laser light coming from dust particles (in dotted region) in $-\hat{z}$ direction. Discharge voltage and gas pressure are set at $370 \mathrm{~V}$ and 0.07 mbar, respectively.

evolution of average intensity profile is shown in the Fig. 5. The red dashed line in the figure indicates the trajectory of a particular crest of the DAW. The phase velocity $\left(v_{p h}\right)$ 
and wavelength $(\lambda)$ are found to be $\sim 2-3 \mathrm{~cm} / \mathrm{sec}$ and $\sim 1.5-2.5 \mathrm{~mm}$, respectively. The wave frequency $\left(f_{d}\right)$ can be estimated from $v_{p h}$ and $\lambda$ and comes out to be $\sim 10-15 \mathrm{~Hz}$. It is also to be noted that the distance between two consecutive crests increases with time as the higher (smaller) amplitude wave moves with faster (slower) velocity ${ }^{14}$. These values of the wave parameters are consistent with the theoretical estimation of dust acoustic waves. For cold dust $\left(T_{d}=0\right)$ and in long wavelength limit $\left(k_{D}>>1\right)$, the phase velocity of dust acoustic wave $v_{p h}=z_{d}\left(k T_{i} n_{d 0} / m_{d} n_{i 0}\right)^{1 / 2}$, where $k, T_{i}, m_{d}, n_{d 0}, n_{i 0}$, and $z_{d}$ are Boltzmann constant, ion temperature, dust particle mass, equilibrium dust density, ion density and dust charge number, respectively ${ }^{40} . \quad z_{d}$ can be estimated by assuming the dust grains are spherical capacitors. Hence, the charge resides on the dust surface can be expressed as $Q_{d}=z_{d} e=4 \pi \varepsilon_{0} r_{d} V_{s}$. The dust surface potential, $V_{s} \sim-4 k T_{e} / e$, is estimated from OML theory $^{1}$. The estimated phase velocity $\left(v_{p h}\right)$ of the DAW is found to be $\sim 3.3 \mathrm{~cm} / \mathrm{sec}$ for parameters $T_{e}=4 \mathrm{eV}, T_{i}=0.025 \mathrm{eV}, m_{d}$ (for an average radius $\left.r_{d} \sim 3 \mu \mathrm{m}\right) \sim 3 \times 10^{-13} \mathrm{~kg}$, $n_{d 0} \sim 1 \times 10^{5} \mathrm{~cm}^{-3}, n_{i 0} \sim 1 \times 10^{9} \mathrm{~cm}^{-3}$, and $z_{d} \sim 3 \times 10^{4}$. Therefore, theoretical predicted value of the phase velocity is in close agreement with the experimental phase velocity of DAWs.

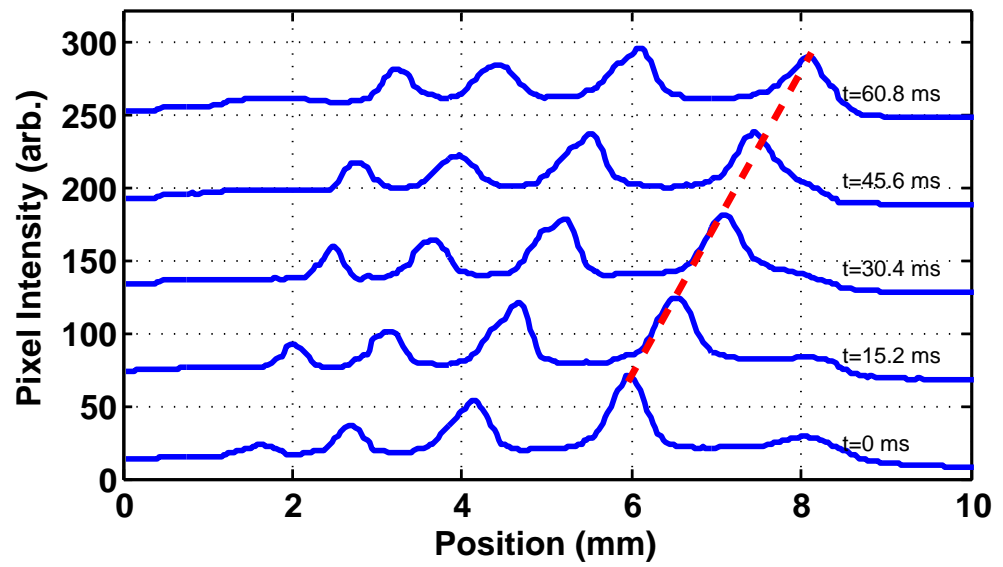

FIG. 5. Time evolution of intensity profile of five video frames taken at time step of $15.2 \mathrm{~ms}$ along the selected region (in Fig. 4(a)) in the vertical plane. Discharge voltage and argon pressure are set at $370 \mathrm{~V}$ and 0.07 mbar respectively for this particular set of experiments

As discussed, the spontaneous DAWs are observed to propagate in the direction of anode to cathode (along the gravity) which essentially in the direction of ion flow. It suggests that the DAW in the present set of experiment could be excited due to ion-dust streaming 
instability. According to earlier investigations ${ }^{26,27}$, in a de discharge dusty plasma, the ion-dust streaming is responsible to excite DAW if ion-drift velocity $\left(u_{i 0}\right)$ becomes in the order or higher than the ion thermal velocity $\left(v_{T i}\right)$ i.e. $u_{i 0} \geq v_{T i}$. In our experiments, the ion thermal speed $v_{T i}=\sqrt{\left(8 k T_{i} / m_{i} \pi\right)} \sim 3.9 \times 10^{4} \mathrm{~cm} / \mathrm{sec}$, where, $m_{i}\left(=6.7 \times 10^{-26} \mathrm{~kg}\right)$ is the mass of argon ions. The ion drift velocity $\left(u_{i 0}\right)$ can be estimated from, $u_{i 0}=\mu_{i} \mathrm{E}$, where $\mu_{i}$ and $\mathrm{E}$ are the ion mobility and the sheath electric field, respectively. The sheath electric field is estimated from the equilibrium condition of dust particles where the gravitational force is exactly balanced by the electrostatic force. Hence at equilibrium, $e z_{d} E=m_{d} g$, where $e\left(=1.6 \times 10^{-19} \mathrm{C}\right)$ is the electronic charge and $g\left(=9.8 \mathrm{~m} / \mathrm{sec}^{2}\right)$ is the acceleration due to gravity. For a given values of $m_{d} \sim 3 \times 10^{-13} \mathrm{~kg}$ and $z_{d} \sim 3 \times 10^{4}$, the calculated electric field $E \sim 6 \mathrm{~V} / \mathrm{cm}$. The mobility of Ar ions $\sim 1.8 \times 10^{3} / p($ torr $) \mathrm{cm}^{2} \mathrm{~s}^{-1} \mathrm{~V}^{-19}$. At $\mathrm{p}=0.07$ mbar, $\mu_{i} \sim 3.5 \times 10^{4} \mathrm{~cm}^{2} \mathrm{~s}^{-1} V^{-1}$. Therefore, the estimated ion-drift velocity, $u_{i 0}$, becomes $\sim 2 \times 10^{5} \mathrm{~cm} / \mathrm{sec}$ (for $\mathrm{E} \sim 6 \mathrm{~V} / \mathrm{cm}$ ). The ratio of $u_{i 0} / v_{T i}$ is $\sim 5$. Hence the above estimation assures that the excitation of DAWs in our experiments is mainly due to the ion-streaming through the dust cloud. It is also found that at higher pressure $(\geq 0.2 \mathrm{mbar})$, the ion-neutral collision frequency increases as a result the oscillations of dust particles get suppressed due to frequent ion-neutral collisions and therefore the waves could not be excited.

\section{CHARACTERISTICS OF DUST ACOUSTIC WAVES IN PRESENCE OF A FLOATING ROD}

\section{A. Vertically aligned floating rod}

After a thorough characterization of Dust Acoustic Wave (DAW), an experiment is carried out to study the modification of its propagation characteristics in presence of a floating object. In this set of experiments, a cylindrical rod is chosen as a floating object and introduced inside the plasma perpendicular to the cathode plane as mentioned in Sec. II. The dimension of the rod is taken in such a way that $r>>\lambda_{D}$. where, $r$ is the radius of the rod and $\lambda_{D} \sim \lambda_{D i}\left(=\sqrt{\epsilon_{0} k T_{i} / n e^{2}}\right)$ is the dust Debye length. In our discharge regimes, $\lambda_{D}$ varies from $0.015 \mathrm{~mm}$ to $0.03 \mathrm{~mm}$. In this regime, it is found that a wire of radius, $r \sim \lambda_{D}$, does not affect (perturb) the dust cloud therefore radius of the rod is chosen bigger 
in dimension $((r=2.5 \mathrm{~mm}))$ so that its influence on DAW can be observed. The effective changes on the dust cloud or DAW in presence of floating cylindrical rod near the upper layer of dust cloud is examined for three different discharge voltages at a particular pressure, $\mathrm{p}=0.07$ mbar. The dust free region (void) around the floating rod is found to be observed at lower discharge voltage where the dust cloud does not exhibit wave structures. This is the case when discharge voltage is $\sim 350 \mathrm{~V}$ at the pressure 0.07 mbar. However, the detail study on dust free region (void) around the floating rod is not the scope of present study and will be reported in future.

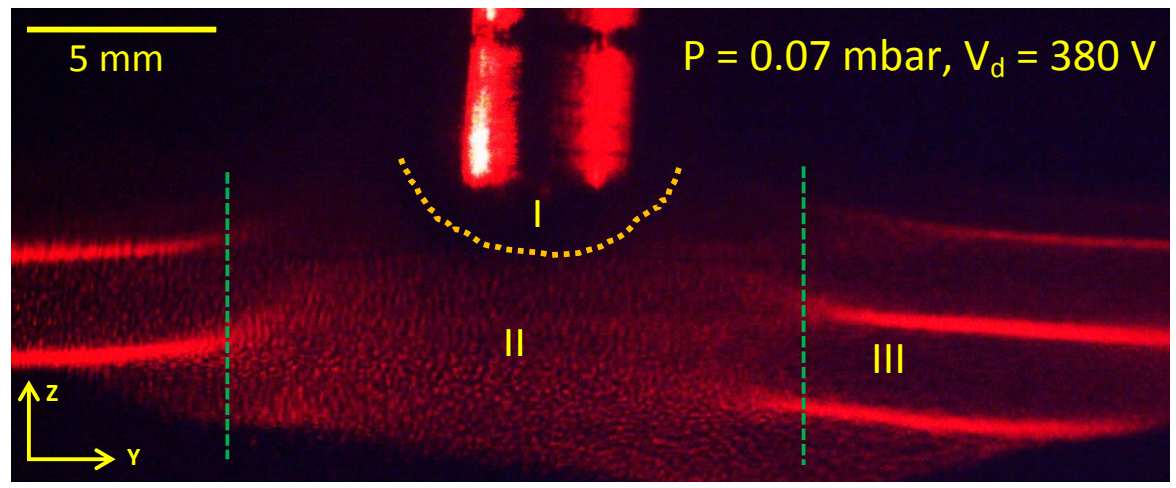

FIG. 6. A video image of dust acoustic waves in presence of a vertically aligned floating rod. Three distinct regions are observed: (I) Dust void (dotted curve). (II) Stationary dust cloud (region between dotted curve and lines). (III). dust acoustic wave (outside the dotted lines). Argon pressure and discharge voltage are set at 0.07 mbar and $380 \mathrm{~V}$, respectively.

At higher discharge voltage (beyond $380 \mathrm{~V}$ ) and the presence of the floating rod (kept far from the dust cloud), the levitated dust cloud exhibits similar kind of propagation of DAWs in the direction of ion flow/gravity as discussed in Sec. V. In this situation, the measured floating potential of the rod which is placed near the upper layer of levitated dust cloud is $\sim-12 \mathrm{~V}$. To study the modification of wave properties, the horizontal (in $\mathrm{X}-\mathrm{Y}$ plane) as well as the vertical (in $\mathrm{Y}-\mathrm{Z}$ plane) slices of images near the floating rod are captured. A typical image (see Fig. 6) of $\mathrm{Y}-\mathrm{Z}$ plane shows the influence of cylindrical rod on the propagation of DAWs. The consecutive frames are used to analyze the dynamical behavior of the dust grains near by the rod. Three distinct regions are found when the rod is brought near the topmost layer of the dust cloud. These regions are classified as: region-I, dust free region near the rod (between rod and dotted curve), region-II, stable dust cloud (between 
the dotted curve and dotted lines) and region-III, dust acoustic wave (outside the dotted lines). The length of dust free region (length from rod surface to dotted curve) is $\sim 2 \mathrm{~mm}$ whereas the length of affected region (length from rod surface to dotted line) is extended upto $\sim 6-7 \mathrm{~mm}$. Outside the dust free region (in region-II), the dust grains only show the random motion instead of participating in the propagation of DAW. The boundary of different regions changes with the change of discharge parameters and the dimension of the rod.

In the presence of a floating rod, the sheath around it modifies the electric field profile of cathode sheath. The resultant electric field is the consequence of the coupling between the sheaths formed around the rod and the cathode, which has been investigated in details by Barnat et al. ${ }^{41}$ in a two component plasma. Hence, as a result the dynamics of ions as well as dust particles get modified in the overlapping sheath region. In the region-I, dust grains are expelled near the rod surface due to the strong sheath electric field which cause the formation of dust void ${ }^{31,32}$. The random motion of dust grains in the region-II is result of the suppression of ion streaming towards the cathode (along the direction of gravity). It is observed that the instabilities in dust cloud are triggered above a critical electric field ${ }^{28}$. In presence of the rod, the electric field becomes weaker ${ }^{41}$ (in the region -II) that causes the suppression of ion-streaming instabilities in the dust cloud and transforms the wave crests into stable dust cloud. In the region-III, the influence of floating rod is negligible therefore the dust cloud exhibits the usual dust acoustic waves.

With the further increase of discharge voltage (beyond $450 \mathrm{~V}$ ), the DAWs become unstable (see Fig. $7(\mathrm{a})$ ) due to the higher value of $E / p$ as reported in $\operatorname{ref}^{26}$ but still propagate in the direction of cathode (along the gravity $(-\hat{z})$ ). With the increase of discharge voltage, the sheath thickness reduces to nearly $28 \mathrm{~mm}$ (as shown in Fig. 3) whereas the sheath electric field increases. Hence the ratio of $E / p$ increases for a given pressure. In this situation, the average wavelength and velocity of DAW are measured as $\sim 2 \mathrm{~mm}$ and $\sim 4 \mathrm{~cm} / \mathrm{sec}$, respectively.

In this particular discharge condition, when the floating rod, with potential $\sim-8 \mathrm{~V}$, is moved from plasma to the upper layer of dust cloud, an unique features in the dust cloud adjacent to the object is observed. A typical image of modified DAW in Y-Z plane $\sim 2 m m$ away from the rod is shown in Fig. 7(b). It is observed that the dust particles are lifted up a height of few mm from the previous equilibrium position (i.e., in absence of the rod) 

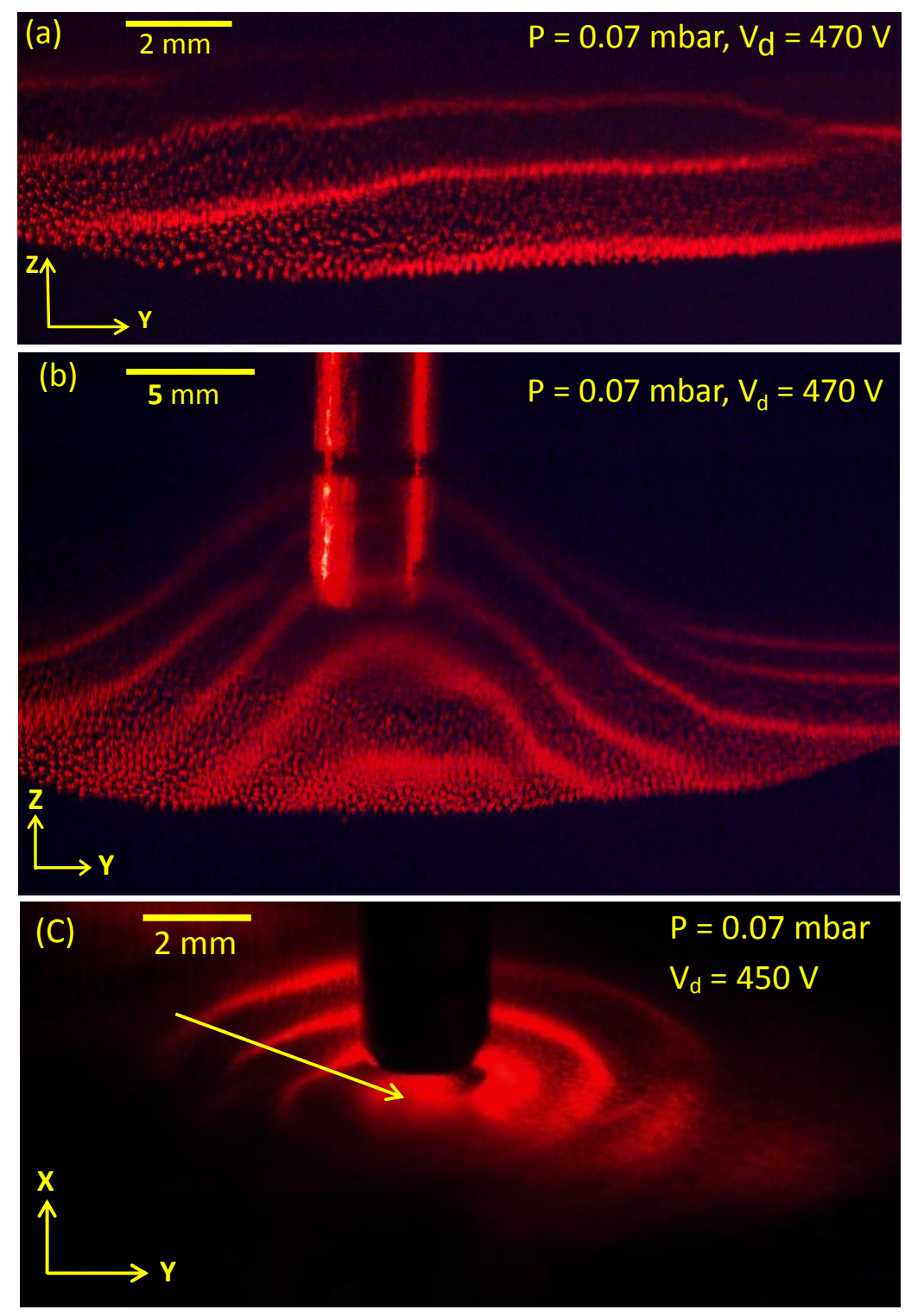

FIG. 7. (a) A video image of dust-acoustic waves in vertical plane (in absence of rod).(b) Image of modified DAWs in vertical $(\mathrm{Y}-\mathrm{Z})$ plane with floating rod where discharge voltage is $470 \mathrm{~V}$. (c) A video image of DAWs in horizontal (radial plane of rod) plane just below the floating rod. Arrow indicates the velocity vector of DAWs in this plane. Argon filling pressure and discharge voltage are set at 0.07 mbar and $450 \mathrm{~V}$, respectively

adjacent to the floating rod as depicted in Fig. 7(b). It means that the dust particles are now levitated at new equilibrium position in presence of the rod and DAWs are originating because of collective oscillatory motions of charged dust particles around their equilibrium 
position. In addition, the linear wavefronts of DAW becomes curved in nature and found to propagate in $\mathrm{Y}-\mathrm{Z}$ plane. It is to be noted that the average wavelength and phase velocity of DAW are $\sim 2.5 \mathrm{~mm}$ and $\sim 3 \mathrm{~cm} / \mathrm{sec}$, respectively. It is worth mentioning that wavelength of DAWs increases whereas the velocity decreases when the rod is brought nearer to the dust cloud. For further characterization of DAWs, the the images are taken in the horizontal $(\mathrm{X}-\mathrm{Y})$ plane. In this plane, the circular wave front of DAWs originates at the outer edge of the perturbed region and propagates in the inward direction (i.e., in the direction of the rod) are observed. A typical video image of DAWs just below the rod in horizontal plane is presented in the Fig. 7(c). Hence, it can be concluded from (Fig. 7(b) and Fig. 7(c)) that the floating rod modifies the propagation characteristics of DAWs which are found to propagates obliquely (along radial $(-\hat{r})$ and gravity $(-\hat{z})$ ).

It is a fact that the dust grains always follow equipotential contours where the electrostatic force acting on them is exactly balanced by the gravitation force. In the presence of a floating rod, the cathode sheath electric field gets modified and dust grains now follow the modified equipotential contour to get an equilibrium position. Hence the dust particles are lifted up a height of few mm adjacent to the rod (in perturbed region). In addition, the dynamics of the ions gets changed due to the change of sheath electric field. Initially (in absence of the rod), the direction of ion streaming is always in $-\hat{z}$ direction whereas in presence of the rod the ion flow direction become oblique, having velocity components along radial $(-\hat{r})$ and gravity $(-\hat{z}))$. As it is discussed in earlier section, the streaming ions is the main responsible cause to excite the dust acoustic waves therefore its propagation direction is changed to oblique.

\section{B. Horizontally aligned floating rod}

To get more insights of the wave-rod interaction, a set of experiments is performed with a horizontally aligned floating rod which is kept always perpendicular to $\mathrm{Y}-\mathrm{Z}$ plane. To study the propagation characteristics of DAWs the images are taken in the $\mathrm{Y}-\mathrm{Z}$ plane for a particular location of X. A series of experiments have been carried out to study the propagation characteristics of DAWs for various discharge voltages $\left(V_{d}\right)$ at a fixed gas pressure (0.07 mbar). It has to be noted that the position of the floating rod is always kept just above the dust cloud (cathode sheath edge) even though the dust cloud position changes with the change of discharge voltage. The change of propagation characteristics of DAWs 
are shown in Fig. 8(a-d) for different discharge voltages. In this range of this discharge voltages, the average wavelength and phase velocity of DAWs also changes from $\sim 2.6-1.7 \mathrm{~mm}$ and $\sim 3.3-4.2 \mathrm{~cm} / \mathrm{sec}$, respectively. These figures clearly indicate the DAWs propagates obliquely with two velocity components (along $\mathrm{Y}$ and $\mathrm{Z}$ ) in the presence of a rod. The $\mathrm{Z}$-component dominates over the $\mathrm{Y}$-component at lower discharge voltage (400 V) whereas $\mathrm{Y}$-component dominates over the $\mathrm{Z}$-component at higher discharge voltage (520 V). The arrows in Fig. 8(a) to Fig. 8(d) indicates the propagation dirction of DAWs which is obtained by analyzing the consecutive frames for different discharge voltages. It is also observed that the dust particles are lifted up a height of few $\mathrm{mm}$ near the rod and this effect becomes significant for higher discharge voltage (Fig. 8(c) Fig. 8(d). Similar to earlier case (with vertical aligned rod), a dust free region always presents near the rod. The volume of the dust free region reduces with the increase of the discharge voltage. It is also found that the dust particles just below the rod always shows random motion at a discharge voltage of $(380-420 \mathrm{~V})$.

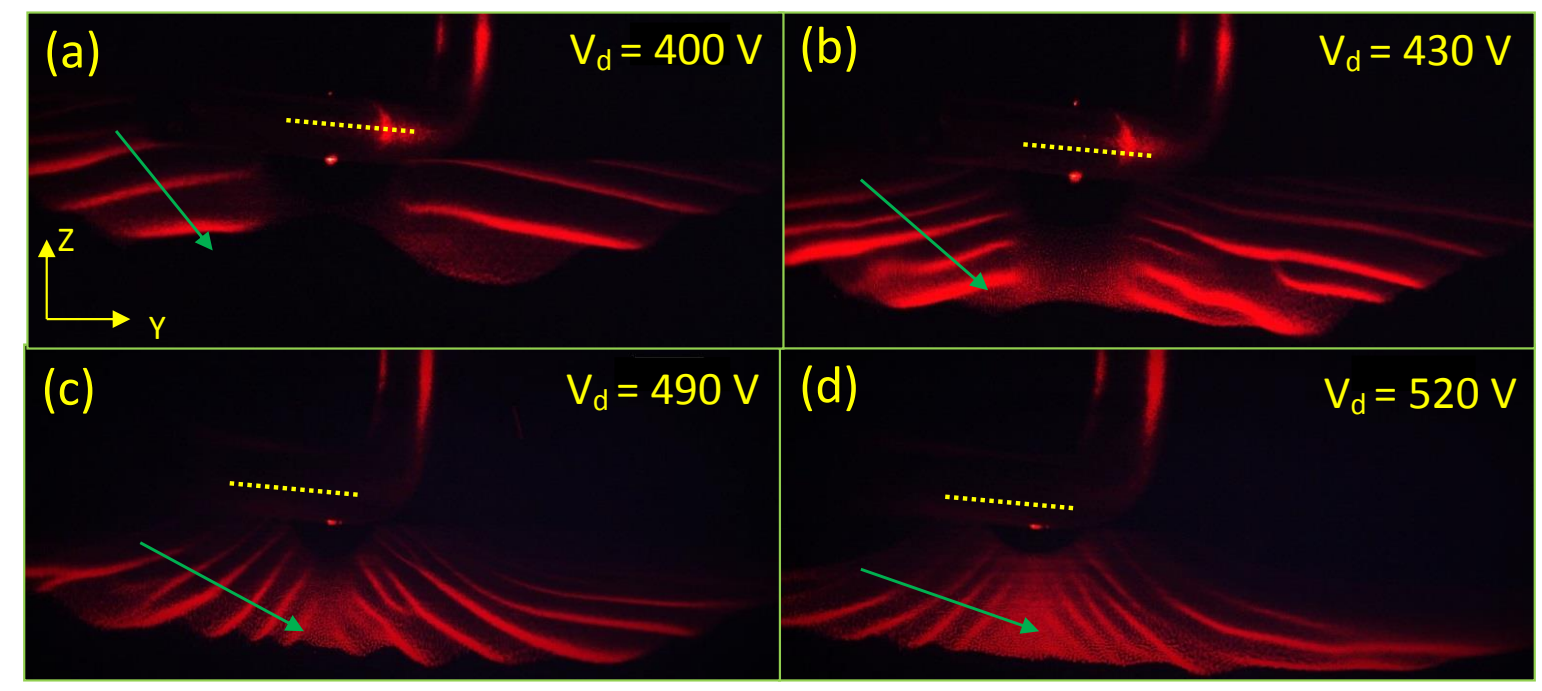

FIG. 8. Video images of dust - acoustic waves with floating rod in horizontal plane: (a) -(d) for discharge voltage $\left(V_{d}\right) 400,430,490$, and $520 \mathrm{~V}$, respectively. The experiments are performed with kaolin particles and gas pressure is set at 0.07 mbar. The yellow dotted lines represent the location of floating rod. Green arrow indicates the direction of the velocity vector with respect to direction of gravity.

In the case of horizontally oriented floating rod, sheath around it interacts with cathode sheath. It is expected that modified (resultant) sheath electric field changes with the change 
of discharge parameters. At lower discharge voltage (400 V), a thick sheath region (strong E-field) around the rod repels the dust grains and dust free region is formed (see Fig. 8(a)). With the increase of discharge voltage (at $520 \mathrm{~V}$ ), the sheath thickness around the rod decreases significantly and as a result the void size reduces (see Fig. 8(d)). The size of dust free region depends on the magnitude of electrostatic and ion-drag force acting on the dust grains. The size of dust void reduces with increasing the ion-drag force compared to the electrostatic force. A Increase of discharge voltage (from $400 \mathrm{~V}$ to $520 \mathrm{~V}$ ) causes a considerable changes in the sheath electric field which results in a significant change of ion dynamics. It is observed that at lower discharge voltage, the velocity component of ions along $\mathrm{Y}$-direction becomes smaller compare to at higher discharge voltage. As a result the DAWs propagates almost in vertical direction (along the gravity) at lower discharge voltage whereas obliquely at higher discharge voltage. This effect is clearly seen in the Fig. 8.

At last, we have further examined the propagation characteristics of DAWs by keeping the floating rod at various Z-locations (measured from cathode surface) for a given discharge condition, $V_{d} \sim 410 \mathrm{~V}$ and $p \sim 0.07$ mbar. Fig. 9(a-d) shows the propagation characteristics of DAWs in presence of this rod for $\mathrm{Z} \sim 32,25,17$ and $10 \mathrm{~mm}$, respectively. It is to be noted that $\mathrm{Z} \sim 32 \mathrm{~mm}$ corresponds to the location of the topmost layer of the dust cloud. As the floating rod is moved from $\mathrm{Z} \sim 32$ to $25 \mathrm{~mm}$, the rod reaches almost to the middle of the dust cloud and modifies the propagation characteristics of DAWs, which is shown in Fig $9(b)$. It is seen in Fig. $9(b)$, a dust free region of length $\sim 2 \mathrm{~mm}$ is formed around the cylindrical rod whereas in the outside of the void region, DAW fronts become concave in nature. It is observed that at $Z \sim 17 \mathrm{~mm}$, the dust cloud takes cone like structure above the floating rod and DAWs propagate obliquely as discussed in earlier section. In the case of Fig $9(d)$, the floating rod is moved deeper to the cathode sheath $(Z \sim 10 \mathrm{~mm})$ where the electric field is most strong compared to three previous locations. In absence of the rod, the dust particle normally could not be found at $\sim 10 \mathrm{~mm}$ whereas in presence of the rod particles are found to exhibit the oscillatory motion. Interestingly at this location, the dust particles follow the floating rod inside the sheath in the form of a cone like structure which is shown in Fig. $9(d)$.

The observed results with floating rod at different locations provide a strong evidence of sheath E-field modification around the floating rod while it interacts with the dust cloud. In Fig. 9(b) (when the rod is kept at the edge of cathode sheath), the variation of E-field 
around the rod is similar to that is reported in the ref ${ }^{41}$. The strong $\mathrm{E}$ - field near the rod pushes the dust grains against ion drag force and as a result dust void is formed. Outside the void region, weak E-field gives rise to the curved shape dust cloud (along with the DAWs). In Fig. 9(c) and 9(d), the cone shaped profile of unstable dust cloud is the result of modified E-field above the $\operatorname{rod}^{41}$. The electric field gets weaker around the floating rod when it is placed inside the sheath region. This lower electric field provides an equllibrium to the dust particles above the rod. Dust Acoustic Waves are found to propagate towards the cathode inside the confined dust cloud.

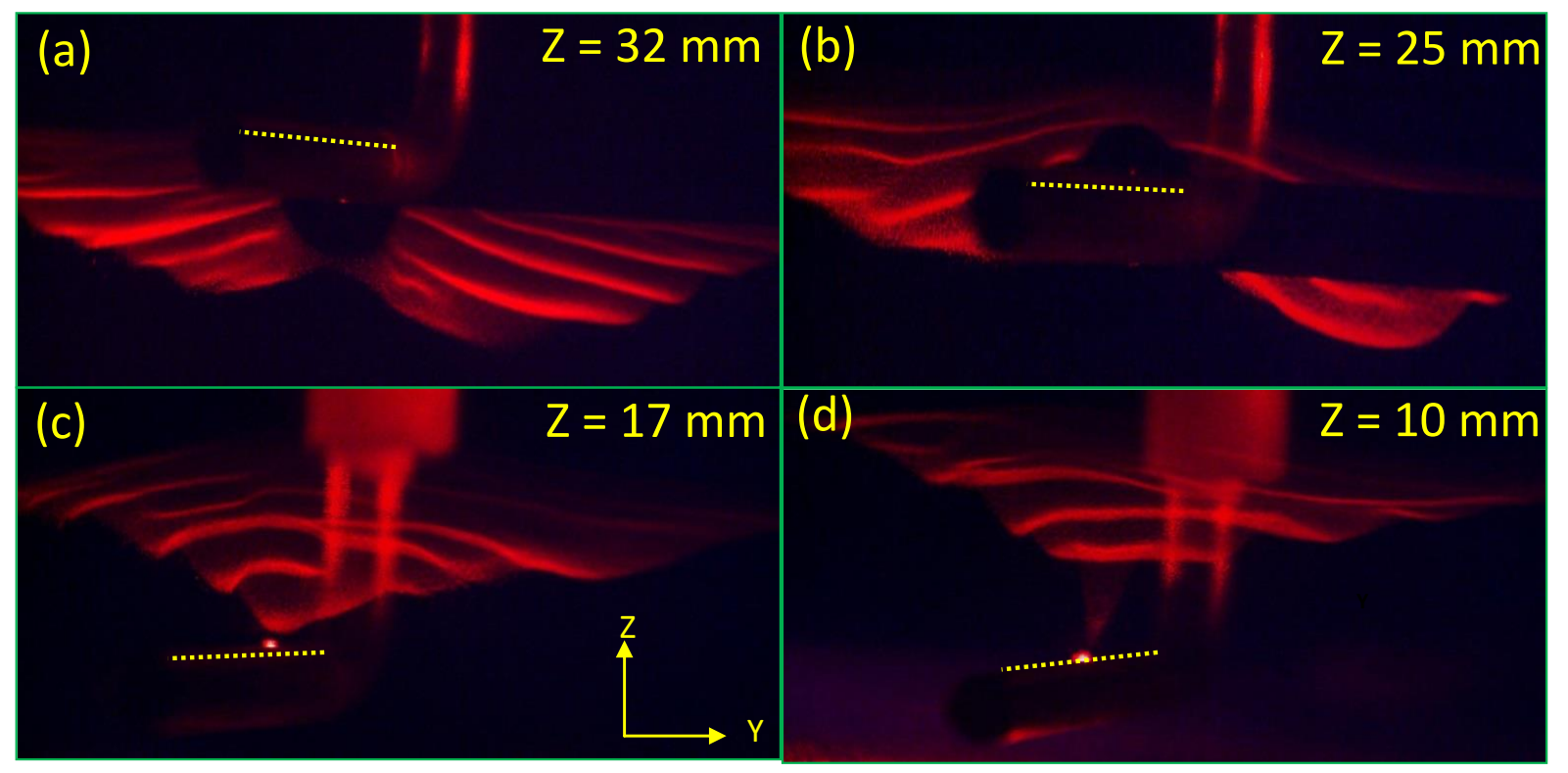

FIG. 9. Video images of DAWs with floating rod at various locations in the sheath region: (a) rod is about near the edge of presheath or sheath. (b) Rod is inside the sheath region where dust particles are levitated. (c) Rod is just below the lower edge of levitated dust cloud. (d) Rod is near the cathode or inside the sheath. The yellow dotted lines represent the location of rod in this plane. The position of floating rod is measured with respect to the cathode surface. Discharge voltage and pressure are set at $410 \mathrm{~V}$ and 0.07 mbar.

\section{SUMMARY AND CONCLUSIONS}

In this paper, we report the experimental observation of self-excited dust acoustic waves and its propagation characteristics in the absence and presence of a floating cylindrical 
object. The DC glow discharge dusty plasma is produced in a glass tube in the background of argon gas. Plasma and dusty plasma parameters are measured/estimated in a wide range of discharge condition. In our experiments, the cathode sheath E-field serves two purposes. Firstly, it provides an electrostatic force that balances the dust particles against gravity. Secondly, the electric field drives the strong downward ion flow which in result excite the DAWs in the dust cloud. A thorough investigation on the propagation of this dust acoustic wave is carried out with and without a floating rod. The main findings of our experimental observations are summarized as follows:

1. Dust acoustic waves are excited spontaneously in an equilibrium dust cloud above a threshold discharge current and below a critical background neutral gas pressure. The low frequency waves are found to propagate along the direction of flow of ions (towards cathode). A detailed characterisation of DAWs are made by measuring the velocity and the wavelength.

2. The propagation characteristics of self-excited DAWs get modified during the interaction of a floating cylindrical object either kept vertically or horizontally. The modification of DAWs in presence of the rod is extended up to $>100 \lambda_{D}$ from the surface of the object.

3. In presence of a floating rod (aligned vertically), the DAWs disappear in the perturbed region at lower discharge voltage $\left(V_{d} \sim 380-400 \mathrm{~V}\right)$ whereas at higher discharge voltage (above $V_{d} \sim 420 \mathrm{~V}$ ), the DAW are found to propagate obliquely. The obliqueness of DAWs changes with the change of discharge parameters.

4. In case of horizontally aligned rod, the DAWs gets excited in a cone shaped dust cloud in the cathode sheath region. Displacement of dust grains in downward (upward) direction confirms that the cathode sheath electric field becomes weaker (stronger) in presence of the floating objects.

The excitation of dust acoustic waves and the modification of its propagation characteristics in presence of a floating object can be explained in terms of streaming ions inside the dust cloud. In absence of the rod, the streaming ions towards the cathode exert a pressure on the levitated dust grains and turns the dust medium into an unstable state, results in the excitation of DAWs which are found to propagate in the direction of gravity. In presence 
of the rod (kept either vertically or horizontally), the sheath around it alters the potential profile of cathode sheath. It happens due to the coupling between the sheaths formed around the cylindrical rod and the cathode electrode. In this coupling, spatial distribution of electric field gets modified in the perturbed region. In this region, dust grains follow the modified equipotential surfaces to get an equilibrium position therefore they are lifted up near the rod. The direction of flow of ions changes according to the sheath electric field and as a results the DAWs are found to propagate obliquely.

\section{ACKNOWLEDGEMENT}

One of the authors M.C. is thankful to Prof. A. Sen, Dr. R. Ganesh and M. Sengupta for their valuable suggestion. The authors are greaful to Dr. M. Bandyopadhyay for his invaluable inputs to improve the manuscript.

\section{REFERENCES}

${ }^{1}$ A. Barkan, N. D'Angelo, and R. L. Merlino, "Charging of dust grains in a plasma," Phys. Rev. Lett. 73, 3093-3096 (1994).

${ }^{2}$ N. N. Rao, P. K. Shukla, and M. Y. Yu, "Dust-acoustic waves in dusty plasmas," Planet. Space Sci. 38, 543-546 (1990).

${ }^{3}$ A. Barkan, R. L. Merlino, and N. D'Angelo, "Laboratory observation of the dust-acoustic wave mode," Phys. Plasmas 2, 3563-3565 (1995).

${ }^{4}$ C. Thompson, A. Barkan, N. D'Angelo, and R. L. Merlino, "Dust acoustic waves in a direct current glow discharge," Phys. Plasmas 4, 2331-2335 (1997).

${ }^{5}$ M. Schwabe, M. Rubin-Zuzic, S. Zhdanov, H. M. Thomas, and G. E. Morfill, "Highly resolved self-excited density waves in a complex plasma," Phys. Rev. Lett. 99, 095002 (2007).

${ }^{6}$ T. M. Flanagan and J. Goree, "Observation of the spatial growth of self-excited dustdensity waves," Phys. Plasmas 17, 123702 (2010).

${ }^{7}$ S. Sarkar, M. Bose, S. Mukherjee, and J. Pramanik, "Spatiotemporal evolution of dielectric driven cogenerated dust density waves," Phys. Plasmas 20, 064502 (2013). 
${ }^{8}$ P. K. Kaw and A. Sen, "Low frequency modes in strongly coupled dusty plasmas," Phys. Plasmas 5, 3552-3559 (1998).

${ }^{9}$ J. Pramanik, G. Prasad, A. Sen, and P. K. Kaw, "Experimental observations of transverse shear waves in strongly coupled dusty plasmas," Phys. Rev. Lett. 88, 175001 (2002).

${ }^{10}$ B. Farokhi, P. K. Shukla, N. L. Tsintsadze, and D. D. Tskhakaya, "Dust lattice waves in a plasma crystal," Phys. Plasmas 7, 814-818 (2000).

${ }^{11}$ A. Homann, A. Melzer, S. Peters, R. Madani, and A. Piel, "Laser-excited dust lattice waves in plasma crystals," Physics Letters A 242, 173 - 180 (1998).

${ }^{12}$ S. Nunomura, D. Samsonov, and J. Goree, "Transverse waves in a two-dimensional screened-coulomb crystal (dusty plasma)," Phys. Rev. Lett. 84, 5141-5144 (2000).

${ }^{13}$ N. N. Rao, "Dust-acoustic kdv solitons in weakly non-ideal dusty plasmas," Phys. Scr. T75, 179-181 (1998).

${ }^{14}$ P. Bandyopadhyay, G. Prasad, A. Sen, and P. K. Kaw, "Experimental study of nonlinear dust acoustic solitary waves in a dusty plasma," Phys. Rev. Lett. 101, 065006 (2008).

${ }^{15}$ Y. Nakamura, H. Bailung, and P. K. Shukla, "Observation of ion-acoustic shocks in a dusty plasma," Phys. Rev. Lett. 83, 1602-1605 (1999).

${ }^{16}$ P. K. Shukla and A. A. Mamun, "Dust-acoustic shocks in a strongly coupled dusty plasma," IEEE Trans. Plasma Sci. 29, 221-225 (2001).

${ }^{17}$ J. Heinrich, S.-H. Kim, and R. L. Merlino, "Laboratory observations of self-excited dust acoustic shocks," Phys. Rev. Lett. 103, 115002 (2009).

${ }^{18}$ V. E. Fortov, O. F. Petrov, V. I. Molotkov, M. Y. Poustylnik, V. M. Torchinsky, V. N. Naumkin, and A. G. Khrapak, "Shock wave formation in a de glow discharge dusty plasma," Phys. Rev. E 71, 036413 (2005).

${ }^{19}$ R. L. Merlino, "25 years of dust acoustic waves," J. Plasma Physics 80, 773-786 (2014).

${ }^{20}$ E. Thomas, Jr, and M. Watson, "First experiments in the dusty plasma experiment device," Phys. Plasmas 6, 4111-4117 (1999).

${ }^{21}$ T. Trottenberg, D. Block, and A. Piel, "Dust confinement and dust-acoustic waves in weakly magnetized anodic plasmas," Phys. Plasmas 13, 042105 (2006).

${ }^{22}$ V. I. Molotkov, O. F. Petrov, M. Y. Pustylnik, V. M. Torchinskii, V. E. Fortov, and A. G. Khrapak, "Dusty plasma of a de glow discharge: methods of investigation and characteristic features of behavior," High Temperature 42, 827-841 (2004). 
${ }^{23}$ J. Pramanik, B. M. Veeresha, G. Prasad, and P. Sen, A.and Kaw, "Experimental observation of dust- acoustic wave turbulence," Physics Letters A 312, 84 - 90 (2003).

${ }^{24}$ M. C. Chang and I. Tsai, Y. Y.and Lin, "Observation of 3d defect mediated dust acoustic wave turbulence with fluctuating defects and amplitude hole filaments," Phys. Plasmas 20, 083703 (2013).

${ }^{25}$ V. E. Fortov, A. D. Usachev, A. V. Zobnin, V. I. Molotkov, and O. F. Petrov, "Dustacoustic wave instability at the diffuse edge of radio frequency inductive low-pressure gas discharge plasma," Phys. Plasmas 10, 1199-1208 (2003).

${ }^{26}$ R. L. Merlino, "Dust-acoustic waves driven by an ion-dust streaming instability in laboratory discharge dusty plasma experiments," Phys. Plasmas 16, 124501 (2009).

${ }^{27}$ V. E. Fortov, A. G. Khrapak, S. A. Khrapak, V. I. Molotkov, A. P. Nefedov, O. F. Petrov, and V. M. Torchinsky, "Mechanism of dust-acoustic instability in a direct current glow discharge plasma," Phys. Plasmas 7, 1374-1380 (2000).

${ }^{28}$ N. D'Angelo and R. L. Merlino, "Current driven dust acoustic instability in a collisional plasma," Planet. Space Sci. 44, 1593 -1598 (1996).

${ }^{29}$ A. A. Mamun and P. K. Shukla, "Streaming instabilities in a collisional dusty plasma," Phys. Plasmas 7, 4412-4417 (2000).

${ }^{30} \mathrm{M}$. Rosenberg, "A note on iondust streaming instability in a collisional dusty plasma," J. Plasma Physics 67, 235-242 (2002).

${ }^{31}$ C. O. Thompson, N. DAngelo, and R. L. Merlino, "The interaction of stationary and moving objects with dusty plasmas," Phys. Plasmas 6, 1421-1426 (1999).

${ }^{32}$ E. Thomas, Jr., K. Avinash, and R. L. Merlino, "Probe induced voids in a dusty plasma," Phys. Plasmas 11, 1770-1774 (2004).

${ }^{33}$ S. Sarkar, M. Mondal, M. Bose, and S. Mukherjee, "Observation of external control and formation of a void in cogenerated dusty plasma," Plasma Sources Sci. Technol. 24, 035007 (2015).

${ }^{34}$ M. Klindworth, A. Piel, A. Melzer, U. Konopka, H. Rothermel, K. Tarantik, and G. E. Morfill, "Dust-free regions around langmuir probes in complex plasmas under microgravity," Phys. Rev. Lett. 93, 195002 (2004).

${ }^{35}$ S.-H. Kim, J. R. Heinrich, and R. L. Merlino, "Diffraction of dust acoustic waves by a circular cylinder," Phys. Plasmas 15, 090701 (2008). 
${ }^{36}$ J. K. Meyer, J. R. Heinrich, S.-H. Kim, and R. L. Merlino, "Interaction of a biased cylinder with a flowing dusty plasma," J. Plasma Physics 79, 677-682 (2013).

${ }^{37}$ D. A. Law, W. H. Steel, B. M. Annaratone, and J. E. Allen, "Probe-induced particle circulation in a plasma crystal," Phys. Rev. Lett. 80, 4189-4192 (1998).

${ }^{38}$ M. Choudhary, S. Mukherjee, and P. Bandyopadhyay, "Transport and trapping of dust particles in a potential well created by inductively coupled diffused plasma." arXiv:1604.03368.

${ }^{39}$ T. E. Sheridan and J. Goree, "Collisional plasma sheath model," Phys.Fluids B 3, 27962804 (1991).

${ }^{40}$ R. L. Merlino, "Dust-acoustic waves: Visible sound waves," AIP Conf. Proc. 1188, 141$152(2009)$.

${ }^{41}$ E. V. Barnat and G. A. Hebner, "Electric field profiles around an electrical probe immersed in a plasma," J. Appl. Phys. 101, 013306 (2007). 